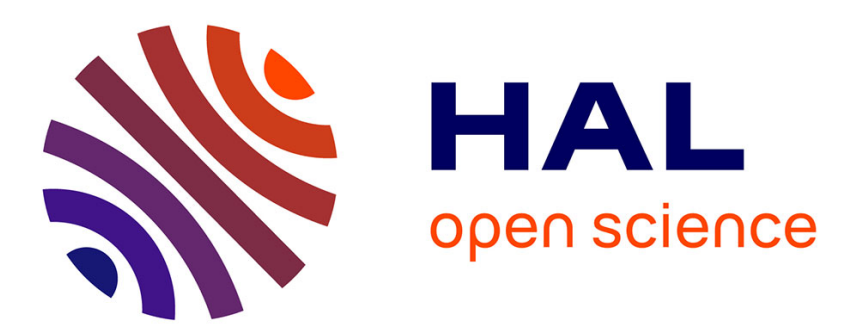

\title{
Adaptive compensation of diffusion-advection actuator dynamics using boundary measurements
}

Delphine Bresch-Pietri, Miroslav Krstic

\section{To cite this version:}

Delphine Bresch-Pietri, Miroslav Krstic. Adaptive compensation of diffusion-advection actuator dynamics using boundary measurements. CDC 2015 - 54th IEEE Conference on Decision and Control, Dec 2015, Osaka, Japan. hal-01251626

\section{HAL Id: hal-01251626 \\ https://hal.science/hal-01251626}

Submitted on 6 Jan 2016

HAL is a multi-disciplinary open access archive for the deposit and dissemination of scientific research documents, whether they are published or not. The documents may come from teaching and research institutions in France or abroad, or from public or private research centers.
L'archive ouverte pluridisciplinaire $\mathbf{H A L}$, est destinée au dépôt et à la diffusion de documents scientifiques de niveau recherche, publiés ou non, émanant des établissements d'enseignement et de recherche français ou étrangers, des laboratoires publics ou privés. 


\title{
Adaptive compensation of diffusion-advection actuator dynamics using boundary measurements
}

\author{
Delphine Bresch-Pietri, Miroslav Krstic
}

\begin{abstract}
For (potentially unstable) Ordinary Differential Equation (ODE) systems with actuator delay, delay compensation can be obtained with a prediction-based control law. In this paper, we consider another class of PDE-ODE cascade, in which the Partial Differential Equation (PDE) accounts for diffusive effects. We investigate compensation of both convection and diffusion and extend a previously proposed control design to handle both uncertainty in the ODE parameters and boundary measurements. Robustness to small perturbations in the diffusion and convection coefficients is also proved.
\end{abstract}

\section{INTRODUCTION}

For linear systems subject to input-delay, prediction-based control strategies, more commonly known as Smith Predictor (see [1],[13],[16]) are state-of-the-art for systems with constant input time-delays (see for instance [2],[3],[5],[9],[14], or [15] and the references therein). Grounding on the use of a prediction of the system state on a time horizon equal to the delay, this technique aims at compensating it, which notably improves transient performances.

This strategy has been analyzed in [10] in the light of distributed parameters systems techniques. In details, representing the delay as a first-order hyperbolic PDE, predictionbased control has been interpreted as the result of a backstepping transformation of the distributed input. This maps the original system into an ODE-PDE cascade in which the output of a finite-time stable transport PDE feeds a stable ODE.

In this work, we propose to study an extension of this technique for diffusion PDE-ODE cascade. Applying the same ideas as in [10] to construct an infinite-dimensional transformation and a corresponding control law, it has been shown in [11] that the closed-loop system can be equivalently represented by a diffusion process, with zero boundary condition, and with the boundary opposite to the controlled one feeding a stable ODE. In this sense, the proposed control law compensates the actuator diffusion. However, while delay dynamics can be finitely stabilized, diffusion compensation should then be understood differently as it introduces an infinite relative degree. Correspondingly, while delay compensation requires to predict future values of the ODE state, in the diffusion case, the control law mainly accounts for an inversion of the diffusion dynamics.

In this work, we are interested in a convection/diffusion PDE-ODE cascade with parameters uncertainties. We pro-

D. Bresch-Pietri (corresponding author) is with CNRS at the Department of Automatic Control, Gipsa-lab, 11 rue des Mathématiques, 38000 Grenoble, France Email: delphine.bresch-pietri@gipsa-lab.fr

M. Krstic is with the Department of Mechanical and Aerospace Engineering, University of California, San Diego, La Jolla CA 92093, USA pose to extend the control law from [11] to the case of boundary measurement and to handle ODE parametric uncertainties in an adaptive manner. We consider that only the ODE state and one boundary condition of the PDE, namely, the control input, are measured. Taking into account the stability of the actuator dynamics, we propose to use a simple openloop observer for the PDE state. Applying classical adaptive techniques [7], [8], [12], we chose the parameter update law grounding on a Lyapunov design and prove closed-loop stability. Finally, we show that the proposed technique is robust to PDE parametric uncertainties. These are the main contributions.

The paper is organized as follows. In Section II, we start by presenting the problem under consideration along with the nominal control design proposed in [11]. Then, in Section III, we detail the proposed boundary adaptive control law and our main result before providing its proof. Robustness to PDE parametric uncertainties is shown in Section IV and illustrated in a numerical example in Section V. We conclude by sketching directions of future work.

Notations. For $u:[0,1] \times \mathbb{R} \rightarrow \mathbb{R}$, we write

$$
\|u(t)\|=\sqrt{\int_{0}^{1} u(x, t)^{2} d x}
$$

\section{Problem Statement}

In this work, we consider the following PDE-ODE cascade

$$
\left\{\begin{aligned}
\dot{X}(t) & =A(\theta) X+B(\theta) u(0, t) \\
u_{t} & =u_{x x}+a u_{x} \\
u_{x}(0, t) & =0 \\
u(1, t) & =U(t)
\end{aligned}\right.
$$

in which $X \in \mathbb{R}^{n}, U$ is scalar, $a>0$ and the dynamics matrices $A$ and $B$ are linearly parametrized with respect to an unknown parameter $\theta \in \mathbb{R}^{p}$, that is,

$$
A(\theta)=A_{0}+\sum_{i=1}^{p} \theta_{i} A_{i} \text { and } B(\theta)=B_{0}+\sum_{i=1}^{p} \theta_{i} B_{i}
$$

As always in indirect adaptive control, certain a priori assumptions on the parameter values are needed in order to ensure stabilizability under parameters estimation. This is why we first characterize further the dynamics under consideration.

Assumption 1: There exists a bounded and known convex set $\Pi=\left\{\boldsymbol{\theta} \in \mathbb{R}^{p} \mid \mathscr{P}(\boldsymbol{\theta}) \leq 0\right\}$, in which the convex function $\mathscr{P}$ is smooth, such that $\theta \in \Pi$. 
Assumption 2: The pair $(A(\theta), B(\theta))$ is controllable for each $\theta \in \Pi$. Furthermore, we assume that there exists a triple of functions $\theta \mapsto(K(\theta), P(\theta), Q(\theta))$ such that $K \in \mathscr{C}^{1}(\Pi)$, $P \in \mathscr{C}^{1}(\Pi), Q \in \mathscr{C}^{0}(\Pi)$, the matrices $P$ and $Q$ are symmetric positive definite and the following equation holds

$$
P(\theta)(A+B K)(\theta)+(A+B K)(\theta)^{T} P(\theta)=-Q(\theta), \quad \theta \in \Pi
$$

To make stabilization possible in the presence of ODE parametric uncertainties [12], we assume that the ODE state is known.

Assumption 3: The state $X$ is measured.

This assumption is standard but quite restrictive. Future works will focus on alleviating it by studying normal ODE forms [12].

In the nominal case, that is, when the system parameters are known and the distributed PDE state $u$ is measured, diffusion can actually be compensated by the controller proposed in [11] in which a purely diffusive process is studied. Furthermore, this control strategy can be extended to dynamics including advection, that is, the case under consideration here, to compensate both convection end diffusion effects, as follows

$$
\begin{aligned}
& U(t)=\int_{0}^{1} k(\theta, 1, x) u(x, t) d x+\gamma(\theta, 1) X(t)
\end{aligned}
$$

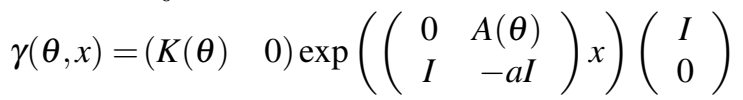

$$
\begin{aligned}
& k(\theta, x, y)=\int_{0}^{x-y} e^{-a(x-y-s)} \gamma(\theta, s) B(\theta) d s
\end{aligned}
$$

In other words, it was shown in [11] (for purely diffusive dynamics, i.e., $a=0$ ) that this control law (5) maps (2) into the target system

$$
\left\{\begin{aligned}
\dot{X}(t) & =(A+B K)(\theta) X(t)+B(\theta) w(0, t) \\
w_{t} & =w_{x x}+a w_{x} \\
w_{x}(0, t) & =0 \\
w(1, t) & =0
\end{aligned}\right.
$$

in which the diffusion and advection effects have been compensated for in the ODE state.

In this paper, we aim at extending this controller to handle (i) ODE parameters uncertainties; and (ii) boundary measurement and control, which means that we assume that the boundary $u(1, t)=U(t)$ is the only element of the PDE state which is measured.

\section{ADAPTIVE BOUNDARY CONTROL DESIGN}

We consider the following open-loop observer

$$
\left\{\begin{aligned}
\hat{u}_{t} & =\hat{u}_{x x}+a \hat{u}_{x} \\
\hat{u}_{x}(0, t) & =0 \\
\hat{u}(1, t) & =U(t)
\end{aligned}\right.
$$

along with the adaptive control law

$$
U(t)=\int_{0}^{1} k(\hat{\theta}, 1, x) \hat{u}(x, t) d x+\gamma(\hat{\theta}, 1) X(t)
$$

which is based on the certainty equivalence principle and involves an estimate $\hat{\theta}$ of the uncertain parameters. Its update law is chosen based on a Lyapunov design (presented in the following section) as

$$
\dot{\hat{\theta}}(t)=\gamma \operatorname{Proj}_{\Pi}\left(\tau_{\theta}(t)\right)
$$

in which the adaptation gain $\gamma$ is a positive constant which has to be chosen small enough (in a sense defined in the sequel) and

$$
\begin{aligned}
\tau_{\theta, i}(t)= & 2\left(X(t)^{T} P(\hat{\theta})+b_{1} \hat{w}_{x}(1, t) \gamma(\hat{\theta}, 1)\right. \\
& \left.-b_{1} \int_{0}^{1}\left[\gamma(\hat{\theta}, x)^{T} \hat{w}(x, t)+\gamma_{x}(\hat{\theta}, x)^{T} \hat{w}_{x}(x, t)\right] d x\right) \\
& \times \frac{A_{i} X(t)+B_{i} \hat{u}(0, t)}{1+N(t)}, \quad i=1, \ldots, p \\
N(t)= & X(t)^{T} P(\hat{\theta}) X(t)+b_{1} \int_{0}^{1}\left(\hat{w}(x, t)^{2}+\hat{w}_{x}(x, t)^{2}\right) d x
\end{aligned}
$$

where the matrix $\mathrm{P}$ is defined in Assumption 2, the function $\gamma$ is given in (6), the constant $b_{1}$ is chosen such that

$$
b_{1}>\frac{4 \max _{\hat{\theta} \in \Pi}|P(\hat{\theta}) B(\hat{\theta})|^{2}}{a \min _{\theta \in \Pi} \min \lambda(Q(\theta))}
$$

the standard projector operator is given by

$$
\begin{aligned}
\operatorname{Proj}_{\Pi}\left\{\tau_{\theta}\right\}= & \hat{\theta} \in \Pi_{\theta} \begin{cases}1, & \text { or } \nabla_{\hat{\theta}} \mathscr{P}^{T} \tau \leq 0 \\
I-\frac{\nabla_{\hat{\theta}} \mathscr{P} \nabla_{\theta} \mathscr{P} T}{\nabla_{\hat{\theta}} \mathscr{P}^{T} \nabla_{\theta} \mathscr{P}} & \hat{\theta} \in \partial \Pi \text { and } \nabla_{\hat{\theta}} \mathscr{P}^{T} \tau>0 .\end{cases}
\end{aligned}
$$

and the distributed variable $\hat{w}$ is defined as

$$
\hat{w}(x, t)=\hat{u}(x, t)-\int_{0}^{x} k(\hat{\theta}, x, y) \hat{u}(y, t) d y-\gamma(\hat{\theta}, x) X(t)
$$

Theorem 1: Let Assumptions 1-3 hold and consider the closed-loop system consisting of (2) with $a>0$, the control law defined through (6)-(7) and (10), and the update law defined by (11)-(13). There exists $\gamma^{*}>0$ such that, for any $\gamma \in\left(0, \gamma^{*}\right)$, there exist positive constants $R_{1}$ and $R_{2}$ such that, for all initial conditions satisfying $\left(X^{0}, u^{0}, \hat{u}^{0}, \hat{\theta}^{0}\right) \in$ $\mathbb{R}^{n} \times H^{2}(0,1) \times H^{2}(0,1) \times \Pi$, the following holds

$$
\Gamma_{1}(t) e^{\Gamma_{2}(t)} \leq R_{1} \Gamma_{1}(0) e^{R_{2} \Gamma_{2}(0)}
$$

where

$$
\begin{aligned}
\Gamma_{1}(t)= & |X(t)|^{2}+\int_{0}^{1} \hat{u}(x, t)^{2} d x+\int_{0}^{1} \hat{u}_{x}(x, t)^{2} d x \\
\Gamma_{2}(t)= & \int_{0}^{1}(u(x, t)-\hat{u}(x, t))^{2} d x+\int_{0}^{1}\left(u_{x}(x, t)-\hat{u}_{x}(x, t)\right)^{2} d x \\
& +|\theta-\hat{\theta}(t)|^{2}
\end{aligned}
$$

Furthermore, both ODE and PDE states asymptotically converge, i.e.,

$$
\begin{gathered}
X(t) \underset{t \rightarrow \infty}{\rightarrow} 0, \quad \max _{x \in[0,1]} u(x, t) \underset{t \rightarrow \infty}{\rightarrow} 0 \\
\text { and } u(1, t)=U(t) \underset{t \rightarrow \infty}{\rightarrow} 0
\end{gathered}
$$




\section{A. Comments}

Before providing the proof of this theorem, we point out a few elements to ease the understanding of this control design.

It is worth underlining the fact that the entire control strategy can be computed with only the measurement of the ODE state and the control law.

As mentioned above, the choice of the update law is based on the Lyapunov design provided below. This explains the appearance of the infinite-dimensional backstepping transformation (16) which is an analysis tool used in the sequel. As common in adaptive control, a projection operator is used in (11) to obtain a physically meaningful value for the parameters estimation. In addition, normalization (13) aims at limiting the rate of change of the parameter estimate, which could otherwise act as a disturbance. This technique is also standard in adaptive control [7], [8], [12] to obtain global stabilization.

As a final remark, notice that the previous design only holds for $a>0$ to allow for the scalar parameter $b_{1}$ to be finite. However, one could straightforwardly modify the stability analysis provided in the sequel when the convection can be neglected compared to the diffusion effect (i.e. $a<1$ ). This should lead to an update law of the form (12) but with a kernel $\cos (x+\alpha)$ under the integrals and a factor $\cos (\alpha)$ multiplying the backstepping variable $\hat{w}_{x}$, in which the kernel parameter $\alpha$ should be chosen into ]0, $\operatorname{arccotan}(a)-1[$. One could infer from the following analysis that the conclusion of Theorem 1 would still hold under similar assumptions but with a lower bound on $b_{1}$ which does not tend to infinity while $a$ tends to zero contrary to (14).

\section{B. Proof of Theorem 1}

We start our analysis by expressing the closed-loop dynamics into the new set of variables $(X, \tilde{u}, \hat{w})$ in which $\tilde{u}=u-\hat{u}$ is the distributed estimation error. First, subtracting (9) to (2) and using the definition of the backstepping transformation (16) along with (6) for $x=0$, one obtains

$$
\left\{\begin{aligned}
\dot{X}(t)= & (A+B K)(\hat{\theta}) X(t)+B(\hat{\theta}) \hat{w}(0, t) \\
& +A(\tilde{\theta}) X(t)+B(\tilde{\theta}) \hat{u}(0, t)+B(\theta) \tilde{u}(0, t) \\
\tilde{u}_{t}= & \tilde{u}_{x x}+a \tilde{u}_{x} \\
\tilde{u}_{x}(0, t)= & 0 \\
\tilde{u}(1, t)= & 0
\end{aligned}\right.
$$

in which we introduced the parameter estimation error $\tilde{\boldsymbol{\theta}}(t)=\theta-\hat{\boldsymbol{\theta}}(t)$ and the matrices

$$
A(\tilde{\theta})=\sum_{i=1}^{p} A_{i} \tilde{\theta}_{i} \text { and } B(\tilde{\theta})=\sum_{i=1}^{p} B_{i} \tilde{\theta}_{i}
$$

Second, taking time- and space-derivatives of (16), one gets

$$
\left\{\begin{aligned}
\hat{w}_{t}= & \hat{w}_{x x}+a \hat{w}_{x}-\dot{\hat{\theta}}(t)^{T} g(x, t)-\gamma(\hat{\theta}, x) A(\tilde{\theta}) X(t) \\
& -\gamma(\hat{\theta}, x)(B \tilde{u}(0, t)+B(\tilde{\theta}) \hat{u}(0, t)) \\
\hat{w}_{x}(0, t)= & 0 \\
\hat{w}(1, t)= & 0
\end{aligned}\right.
$$

with

$$
g(x, t)=\int_{0}^{x} k_{\hat{\theta}}(\hat{\boldsymbol{\theta}}, x, y) \hat{u}(y, t) d y+\gamma_{\hat{\boldsymbol{\theta}}}(\hat{\boldsymbol{\theta}}, x) X(t)
$$

in which $k_{\hat{\theta}}$ and $\gamma_{\hat{\theta}}$ are continuous functions from the definitions (6)-(7) and Assumption 2. In the sequel, we also need the governing equation of the spatial-derivative of these distributed variables which can be obtained straightforwardly as

$$
\left\{\begin{array}{l}
\tilde{u}_{x t}=\tilde{u}_{x x x}+a \tilde{u}_{x x} \\
\tilde{u}_{x x}(0, t)=\tilde{u}_{t}(0, t) \\
\tilde{u}_{x}(1, t)=-\frac{1}{a} \tilde{u}_{x x}(1, t)
\end{array}\right.
$$

and

$$
\left\{\begin{array}{c}
\hat{w}_{x t}=\hat{w}_{x x x}+a \hat{w}_{x x}-\dot{\hat{\theta}}(t)^{T} g_{x}(x, t)-\gamma_{x}(\hat{\theta}, x) A(\tilde{\theta}) X(t) \\
-\gamma_{x}(\hat{\theta}, x)(B \tilde{u}(0, t)+B(\tilde{\theta}) \hat{u}(0, t)) \\
\hat{w}_{x x}(0, t)=\hat{w}_{t}(0, t) \\
\hat{w}_{x}(1, t)=\frac{1}{a}\left(-\hat{w}_{x x}(1, t)+\dot{\hat{\theta}}^{T} g(1, t)+\gamma(\hat{\theta}, 1) A(\tilde{\theta}) X(t)\right. \\
+\gamma(\hat{\theta}, 1)(B(\tilde{\theta}) \hat{u}(0, t)+B \tilde{u}(0, t))
\end{array}\right.
$$

We are now ready to carry out the Lyapunov analysis. Consider the following Lyapunov functional candidate

$$
\begin{aligned}
V(t)= & \log (1+N(t))+b_{2} \int_{0}^{1} \tilde{u}(x, t)^{2} d x \\
& +b_{2} \int_{0}^{1} \tilde{u}_{x}(x, t)^{2} d x+\frac{1}{2 \gamma}|\tilde{\theta}(t)|^{2}
\end{aligned}
$$

in which $N$ has been introduced in (13), $b_{2}$ is a positive constant and $\gamma$ and $b_{1}$ are the tuning parameters involved in the update law (11). Taking a time-derivative and using (21)(26) and integrations by parts, one gets

$$
\begin{aligned}
& \dot{V}(t)=\frac{1}{1+N(t)}\left(-X(t)^{T} Q(\hat{\theta}) X(t)+2 X(t) P(\hat{\theta})(B(\hat{\theta}) \hat{w}(0, t)\right. \\
& +A(\tilde{\theta}) X(t)+B(\tilde{\theta}) \hat{u}(0, t)+B(\theta) \tilde{u}(0, t))-2 b_{1}\left\|\hat{w}_{x}(t)\right\|^{2} \\
& -a b_{1} \hat{w}(0, t)^{2}-2 b_{1}\left\|\hat{w}_{x x}(t)\right\|^{2}-a b_{1} \hat{w}_{x}(1, t)^{2}+2 b_{1} \hat{w}_{x}(1, t) \\
& \times\left(\hat{\hat{\theta}}(t)^{T} g(1, t)+\gamma(\hat{\theta}, 1)(A(\tilde{\theta}) X(t)+B(\tilde{\theta}) \hat{u}(0, t)+B \tilde{u}(0, t))\right. \\
& -2 b_{1} \dot{\hat{\theta}}(t)^{T} \int_{0}^{1}\left(g(x, t) \hat{w}(x, t)+g_{x}(x, t) \hat{w}_{x}(x, t)\right) d x \\
& -2 b_{1} \int_{0}^{1} \gamma(\hat{\theta}, x)(A(\tilde{\theta}) X(t)+B \tilde{u}(0, t)+B(\tilde{\theta}) \hat{u}(0, t)) \hat{w}(x, t) d x \\
& -2 b_{1} \int_{0}^{1} \gamma_{x}(\hat{\theta}, x)(A(\tilde{\theta}) X(t)+B \tilde{u}(0, t)+B(\tilde{\theta}) \hat{u}(0, t)) \hat{w}_{x}(x, t) d x \\
& \left.-\sum_{i=1}^{p} \dot{\hat{\theta}}_{i}(t)^{T} X(t)^{T} \frac{d P}{d \hat{\theta}_{i}}(\hat{\theta}) X(t)\right)-a b_{2} \tilde{u}(0, t)^{2}-2 b_{2}\left\|\tilde{u}_{x}(t)\right\|^{2} \\
& -a b_{2} \tilde{u}_{x}(1, t)^{2}-2 b_{2}\left\|\tilde{u}_{x x}(t)\right\|^{2}-\frac{1}{\gamma} \tilde{\theta}^{T} \dot{\hat{\theta}}(t)
\end{aligned}
$$

Using the projection operator properties, one obtains

$$
\begin{aligned}
\dot{V}(t) & \leq \frac{1}{1+N(t)}\left(-\underline{\lambda}|X(t)|^{2}+2 X(t) P(\hat{\theta})(B(\hat{\boldsymbol{\theta}}) \hat{w}(0, t)\right. \\
& +B(\theta) \tilde{u}(0, t))-2 b_{1}\left\|\hat{w}_{x}(t)\right\|^{2}-a b_{1} \hat{w}(0, t)^{2} \\
& -2 b_{1}\left\|\hat{w}_{x x}(t)\right\|^{2}-a b_{1} \hat{w}_{x}(1, t)^{2} \\
& +2 b_{1} \hat{w}_{x}(1, t)\left(\hat{\hat{\theta}}(t)^{T} g(1, t)+\gamma(\hat{\theta}, 1) B \tilde{u}(0, t)\right) \\
& -2 b_{1} \int_{0}^{1}\left[\dot{\hat{\theta}}(t)^{T} g(x, t)+\gamma(\hat{\boldsymbol{\theta}}, x) B \tilde{u}(0, t)\right] \hat{w}(x, t) d x \\
& -2 b_{1} \int_{0}^{1}\left[\dot{\hat{\theta}}(t)^{T} g_{x}(x, t)+\gamma_{x}(\hat{\boldsymbol{\theta}}, x) B \tilde{u}(0, t)\right] \hat{w}_{x}(x, t) d x
\end{aligned}
$$




$$
\begin{aligned}
& \left.+\max _{1 \leq i \leq p, \hat{\theta} \in \Pi}\left|\frac{d P}{d \hat{\theta}_{i}}(\hat{\theta})\right| \sum_{i=1}^{p} \dot{\hat{\theta}}_{i}(t)^{T}|X(t)|^{2}\right)-a b_{2} \tilde{u}(0, t)^{2} \\
& -2 b_{2}\left\|\tilde{u}_{x}(t)\right\|^{2}-a b_{2} \tilde{u}_{x}(1, t)^{2}-2 b_{2}\left\|\tilde{u}_{x x}(t)\right\|^{2}
\end{aligned}
$$

in which $\underline{\lambda}=\min _{\theta \in \Pi} \min \lambda(Q(\theta))>0$. Taking into account the fact that the inverse of the backstepping transformation (16) is (see [11], [17])

$$
\hat{u}(x, t)=\hat{w}(x, t)+\int_{0}^{x} \ell(\hat{\boldsymbol{\theta}}, x, y) \hat{w}(y, t) d y+\beta(\hat{\boldsymbol{\theta}}, x) X(t)
$$

with

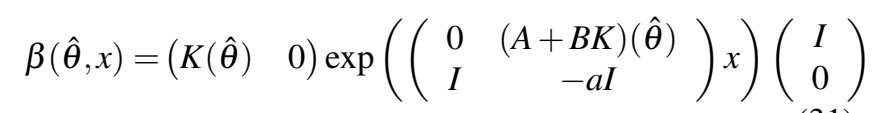$$
\ell(\hat{\boldsymbol{\theta}}, x, y)=\int_{0}^{x-y} e^{-a(x-y-s)} \beta(\hat{\boldsymbol{\theta}}, s) B(\hat{\boldsymbol{\theta}}) d s
$$

one can observe, with the help of Young and CauchySchwartz inequalities, that there exists $M>0$ such that

$$
\begin{aligned}
& 2 \hat{w}_{x}(1, t) \dot{\hat{\theta}}(t)^{T} g(1, t) \leq \gamma M \hat{w}_{x}(1, t)^{2} \\
& 2 \hat{w}_{x}(1, t) \gamma(\hat{\theta}, 1) B \tilde{u}(0, t) \leq \frac{a}{2} \hat{w}_{x}(1, t)^{2}+M \tilde{u}(0, t)^{2} \\
&\left|\hat{\theta}_{i}(t)\right| \leq \gamma M, \quad i=1, \ldots, p \\
& 2|\dot{\hat{\theta}}(t)|\left(\left|\int_{0}^{1} g(x, t) \hat{w}(x, t) d x\right|+\left|\int_{0}^{1} g_{x}(x, t) \hat{w}_{x}(x, t) d x\right|\right) \\
& \leq 2 M \gamma\left(|X(t)|^{2}+\|\hat{w}(t)\|^{2}+\left\|\hat{w}_{x}(t)\right\|^{2}\right) \\
& 2\left|\int_{0}^{1} \gamma(\hat{\theta}, x) B \tilde{u}(0, t) \hat{w}(x, t) d x\right| \leq \frac{\|\hat{w}(t)\|^{2}}{8}+M \tilde{u}(0, t)^{2} \\
& 2\left|\int_{0}^{1} \gamma_{x}(\hat{\theta}, x) B \tilde{u}(0, t) \hat{w}_{x}(x, t) d x\right| \leq \frac{\left\|\hat{w}_{x}(t)\right\|^{2}}{2}+M \tilde{u}(0, t)^{2}
\end{aligned}
$$

Using those inequalities and the fact that $N(t)>0$, one gets

$$
\begin{aligned}
\dot{V}(t) & \leq \frac{1}{1+N(t)}\left(-\left(a b_{1}-\frac{4}{\underline{\lambda}}|P(\hat{\theta}) B(\hat{\theta})|^{2}\right) \hat{w}(0, t)^{2}\right. \\
& -\left(\frac{\lambda}{2}-\gamma M\left(p \max _{1 \leq i \leq p, \hat{\theta} \in \Pi}\left|\frac{d P}{d \hat{\theta}_{i}}(\hat{\theta})\right|+2\right)\right)|X(t)|^{2} \\
& -\left(\frac{b_{1}}{8}-2 \gamma M\right)\left(\|\hat{w}(t)\|^{2}+\left\|\hat{w}_{x}(t)\right\|^{2}\right) \\
& \left.-b_{1}\left\|\hat{w}_{x x}(t)\right\|^{2}-\left(\frac{a b_{1}}{2}-\gamma M\right) \hat{w}_{x}(1, t)^{2}\right) \\
& -\frac{b_{2}}{4}\left(\|\tilde{u}(t)\|^{2}+\left\|\tilde{u}_{x}(t)\right\|^{2}\right)-a b_{2} \tilde{u}_{x}(1, t)^{2}-2 b_{2}\left\|\tilde{u}_{x x}(t)\right\|^{2} \\
& -\left(a b_{2}-3 b_{1} M-\frac{4}{\lambda}|P(\hat{\theta}) B(\theta)|^{2}\right) \tilde{u}(0, t)^{2}
\end{aligned}
$$

in which we have also used the Poincaré inequality (taking advantage of the fact that $\hat{w}(1, t)=0)$

$$
\|\hat{w}(t)\|^{2} \leq 4\left\|\hat{w}_{x}(t)\right\|^{2}
$$

and a similar one for the distributed estimation error $\tilde{u}$. Consequently, by choosing $b_{1}$ such that (14) holds and

$$
b_{2}>\frac{1}{a}\left(3 b_{1} M-\frac{4}{\underline{\lambda}} \max _{\hat{\theta} \in \Pi}|P(\hat{\theta}) B(\hat{\theta})|^{2}\right)
$$

$$
\begin{aligned}
& \gamma<\gamma^{*}
\end{aligned}
$$

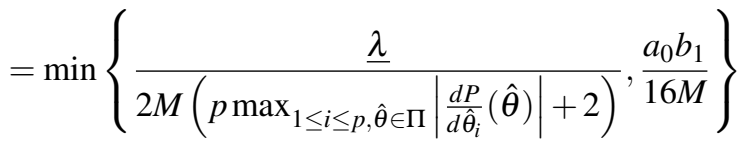

in which $a_{0}=\min \{a, 1\}$, one finally obtains

$$
V(t) \leq V(0), \quad t \geq 0 .
$$

Using (16) and its inverse (16), one can show that there exist strictly positive constants $c_{1}, c_{2}, c_{3}$ and $c_{4}$ such that

$$
\begin{aligned}
\|\hat{u}(t)\|^{2} & \leq c_{1}\left(|X(t)|^{2}+\|\hat{w}(t)\|^{2}\right) \\
\|\hat{w}(t)\|^{2} & \leq c_{2}\left(|X(t)|^{2}+\|\hat{u}(t)\|^{2}\right) \\
\left\|\hat{u}_{x}(t)\right\|^{2} & \leq c_{3}\left(|X(t)|^{2}+\|\hat{w}(t)\|^{2}+\left\|\hat{w}_{x}(t)\right\|^{2}\right) \\
\left\|\hat{w}_{x}(t)\right\|^{2} & \leq c_{4}\left(|X(t)|^{2}+\|\hat{u}(t)\|^{2}+\left\|\hat{u}_{x}(t)\right\|^{2}\right)
\end{aligned}
$$

and (17) follows from there.

This last inequality guarantees that $|X|,\|\hat{w}\|,\left\|\hat{w}_{x}\right\|,\|\tilde{u}\|$, $\left\|\tilde{u}_{x}\right\|$ and $|\tilde{\theta}|$ are uniformly bounded. Now, integrating (39) between zero and infinity, one obtains that $X,\|\hat{w}\|$ and $\left\|\hat{w}_{x}\right\|$ are square integrable. Hence, from the following Agmon inequality,

$$
\max _{x \in[0,1]} \hat{w}(x, t)^{2} \leq 2\|\hat{w}(t)\|\left\|\hat{w}_{x}(t)\right\|
$$

which uses the fact that $\hat{w}(1, t)=0$, one infers that $\hat{w}(x, \cdot)$ is also square integrable and uniformly bounded for all $x \in[0,1]$. Thus, using (44),(46) and the backstepping definition (16), the same properties hold for $\|\hat{u}\|,\left\|\hat{u}_{x}\right\|$ and $\hat{u}(x, \cdot)$ for all $x \in[0,1]$. Similarly, the same properties hold for $\|\tilde{u}\|,\left\|\tilde{u}_{x}\right\|$ and $\tilde{u}(x, \cdot)$ for all $x \in[0,1]$ and thus for $\|u\|,\left\|u_{x}\right\|$ and $u(x, \cdot)$ for all $x \in[0,1]$ by definition of $\tilde{u}$. Further,

$$
\begin{aligned}
\frac{d}{d t}|X(t)|^{2}= & 2 X(t)^{T}(A X(t)+B u(0, t)) \\
\frac{d}{d t}\|\hat{w}(t)\|^{2}= & -2\left\|\hat{w}_{x}(t)\right\|^{2}-\hat{w}(0, t)^{2}-2 b_{1} \int_{0}^{1} \gamma(\hat{\theta}, x) \\
& \times(A(\tilde{\theta}) X(t)+B \tilde{u}(0, t)+B(\tilde{\theta}) \hat{u}(0, t)) \hat{w}(x, t) d x \\
& -2 b_{1} \hat{\hat{\theta}}(t)^{T} \int_{0}^{1} g(x, t) \hat{w}(x, t) d x \\
\frac{d}{d t}\|\tilde{u}(t)\|^{2}= & -2\left\|\tilde{u}_{x}(t)\right\|^{2}-\tilde{u}(0, t)^{2}
\end{aligned}
$$

in which the right-hand terms are bounded applying Young and Cauchy-Schwartz inequality and the previous considerations. Applying Barbalat lemma, it follows that $|X|,\|\tilde{u}\|$ and $\|\hat{w}\|$ converge to zero as $t$ tends to infinity. Using Agmon inequality, one obtains that $\max _{x \in[0,1]} \tilde{u}(x, \cdot)$ and $\max _{x \in[0,1]} \hat{w}(x, \cdot)$ also converge to zero, as $\left\|\tilde{u}_{x}\right\|$ and $\|\hat{w}\|$ are bounded. From there, using the inverse backstepping transformation (30), one concludes that $\max _{x \in[0,1]} u(x, \cdot)$ converge to zero which concludes the proof.

\section{RoBUSTNESS TO DIFFUSION/ADVECTION PARAMETERS UNCERTAINTY}

In this section, we study robustness of the following closed loop system, in which $U(t)$ is given in (10),

$$
\left\{\begin{aligned}
\dot{X}(t) & =A(\theta) X(t)+B(\theta) u(0, t) \\
u_{t} & =\left(1+\varepsilon_{1}\right) u_{x x}+a\left(1+\varepsilon_{2}\right) u_{x} \\
u_{x}(0, t) & =0 \\
u(1, t) & =U(t)
\end{aligned}\right.
$$


with respect to the parameters $\varepsilon_{1}$ and $\varepsilon_{2}$ which are assumed to be either positive of negative but small a priori. We have the following result.

Theorem 2: Let Assumptions 1-3 hold and consider the closed-loop system consisting of the dynamics (52), the controller defined through (6)-(7) and (10), and the update law defined by (11)-(13). Consider also the functionals $\Gamma_{1}$ and $\Gamma_{2}$ introduced in (18). There exist decreasing functions $\varepsilon_{1}^{*}, \varepsilon_{2}^{*}: \mathbb{R}^{2} \rightarrow \mathbb{R}^{*}$ such that, if $\left|\varepsilon_{1}\right|<\varepsilon_{1}^{*}\left(\Gamma_{1}(0), \Gamma_{2}(0)\right)$ and $\left|\varepsilon_{2}\right|<\varepsilon_{2}^{*}\left(\Gamma_{1}(0), \Gamma_{2}(0)\right)$, then the conclusions of Theorem 1 still hold.

Proof: Using the inverse backstepping transformation (30), the error system now writes

$$
\left\{\begin{array}{l}
\tilde{u}_{t}=\left(1+\varepsilon_{1}\right) \tilde{u}_{x x}+a\left(1+\varepsilon_{2}\right) \tilde{u}_{x}+\varepsilon_{1} g_{1}(x, t)+a \varepsilon_{2} g_{2}(x, t) \\
\tilde{u}_{x}(0, t)=0 \\
\tilde{u}(1, t)=0
\end{array}\right.
$$

in which

$$
\begin{aligned}
g_{1}(x, t)= & \hat{w}_{x x}(x, t)+K B(\hat{\theta}) \hat{w}(x, t)+\int_{0}^{x} \ell_{x x}(\hat{\theta}, x, y) \hat{w}(y, t) d y \\
& +\beta(\hat{\theta}, x)(A+B K)(\hat{\theta}) X(t)-a \beta_{x}(\hat{\theta}, x) X(t) \\
g_{2}(x, t)= & \hat{w}_{x}(x, t)+\int_{0}^{x} \ell_{x}(\hat{\theta}, x, y) \hat{w}(y, t)+\beta_{x}(\hat{\theta}, x) X(t)
\end{aligned}
$$

and its space-derivative satisfies

$$
\left\{\begin{aligned}
\tilde{u}_{x t}=\quad & \left(1+\varepsilon_{1}\right) \tilde{u}_{x x x} \\
& +a\left(1+\varepsilon_{2}\right) \tilde{u}_{x x}+\varepsilon_{1} g_{1, x}(x, t)+a \varepsilon_{2} g_{2, x}(x, t) \\
\tilde{u}_{x}(1, t)= & -\frac{1}{a\left(1+\varepsilon_{2}\right)}\left(\left(1+\varepsilon_{1}\right) \tilde{u}_{x x}(1, t)\right. \\
& \left.+\varepsilon_{1} g_{1}(1, t)+a \varepsilon_{2} g_{2}(1, t)\right)
\end{aligned}\right.
$$

Thus, using the following integration by parts,

$$
\int_{0}^{1} \tilde{u}_{x}(x, t) \hat{w}_{x x x}(x, t) d x=\tilde{u}_{x}(1, t) \hat{w}_{x x}(1, t)+\int_{0}^{1} \tilde{u}_{x x} \hat{w}_{x x}(x, t) d x
$$

and using the same techniques as in the previous section, one obtains the existence of a constant $M_{0}>0$ such that

$$
\begin{aligned}
& \frac{d}{d t}\left(\|\tilde{u}(t)\|^{2}+\left\|\tilde{u}_{x}(t)\right\|^{2}\right) \leq-2\left(1+\varepsilon_{1}\right)\left(\left\|\tilde{u}_{x}(t)\right\|^{2}+\left\|\tilde{u}_{x x}(t)\right\|^{2}\right) \\
& \quad-a\left(1+\varepsilon_{2}\right) \tilde{u}(0, t)^{2}-a\left(1+\varepsilon_{2}\right) \tilde{u}_{x}(1, t)^{2}+M_{0}\left(\left|\varepsilon_{1}\right|+\left|\varepsilon_{2}\right|\right) \\
& \quad \times\left(|X(t)|^{2}+\|\hat{w}(t)\|^{2}+\hat{w}(0, t)^{2}+\left\|\hat{w}_{x}(t)\right\|^{2}+\hat{w}_{x}(1, t)^{2}\right. \\
& \quad+\left\|\hat{w}_{x x}(t)\right\|^{2}+\|\tilde{u}(t)\|^{2}+\tilde{u}(0, t)^{2}+\left\|\tilde{u}_{x}(t)\right\|+\tilde{u}_{x}(1, t)^{2} \\
& \left.\quad+\left\|\tilde{u}_{x x}(t)\right\|^{2}\right)
\end{aligned}
$$

Hence, equations (29) and (39) given in the proof of Theorem 1 now rewrite as

$$
\begin{aligned}
\dot{V}(t) & \leq \frac{1}{1+N(t)}\left(-\left(a b_{1}-\frac{4}{\underline{\lambda}}|P(\hat{\theta}) B(\hat{\theta})|^{2}\right) \hat{w}(0, t)^{2}\right. \\
& -\left(\frac{\lambda}{2}-\gamma M\left(p \max _{1 \leq i \leq p, \hat{\theta} \in \Pi}\left|\frac{d P}{d \hat{\theta}_{i}}(\hat{\theta})\right|+2\right)\right)|X(t)|^{2} \\
& -\left(\frac{b_{1}}{8}-2 \gamma M\right)\left(\|\hat{w}(t)\|^{2}+\left\|\hat{w}_{x}(t)\right\|^{2}\right) \\
& \left.-b_{1}\left\|\hat{w}_{x x}(t)\right\|-\left(\frac{a b_{1}}{2}-\gamma M\right) \hat{w}_{x}(1, t)^{2}\right)
\end{aligned}
$$

$$
\begin{aligned}
& -\frac{b_{2}}{4}\left(\|\tilde{u}(t)\|^{2}+\left\|\tilde{u}_{x}(t)\right\|^{2}\right)-a b_{2} \tilde{u}_{x}(1, t)^{2}-2 b_{2}\left\|\tilde{u}_{x x}(t)\right\|^{2} \\
& -\left(a b_{2}-3 b_{1} M-\frac{4}{\underline{\lambda}}|P(\hat{\theta}) B(\theta)|^{2}\right) \tilde{u}(0, t)^{2} \\
& +b_{2}\left(M_{0}+2+a\right)\left(\left|\varepsilon_{1}\right|+\left|\varepsilon_{2}\right|\right)\left(|X(t)|^{2}+\|\hat{w}(t)\|^{2}+\hat{w}(0, t)^{2}\right. \\
& +\left\|\hat{w}_{x}(t)\right\|^{2}+\hat{w}_{x}(1, t)^{2}+\left\|\hat{w}_{x x}(t)\right\|^{2}+\|\tilde{u}(t)\|^{2} \\
& +\tilde{u}(0, t)^{2}+\left\|\tilde{u}_{x}(t)\right\|+\tilde{u}_{x}(1, t)^{2}+\left\|\tilde{u}_{x x}(t)\right\|^{2}
\end{aligned}
$$

Define

$$
\begin{aligned}
& V_{1}(t)=|X(t)|^{2}+\|\hat{w}(t)\|^{2}+\left\|\hat{w}_{x}(t)\right\|^{2} \\
& V_{2}(t)=\hat{w}(0, t)^{2}+\hat{w}_{x}(1, t)^{2}+\left\|\hat{w}_{x x}(t)\right\|^{2} \\
& V_{3}(t)=\|\tilde{u}(t)\|^{2}+\left\|\tilde{u}_{x}(t)\right\|^{2} \\
& V_{4}(t)=\tilde{u}(0, t)^{2}+\tilde{u}_{x}(1, t)^{2}+\left\|\tilde{u}_{x x}(t)\right\|^{2} \\
& \Upsilon_{1}(t)=\Gamma_{1}(t)+\left\|\hat{w}_{x x}(t)\right\|^{2} \\
& \Upsilon_{2}(t)=\Gamma_{2}(t)+\left\|\tilde{u}_{x x}(t)\right\|^{2}
\end{aligned}
$$

Under the conditions (14), (41)-(42), there exist $\eta_{1}, \eta_{2}>0$ such that (58) rewrites

$$
\begin{aligned}
\dot{V}(t) & \leq-\frac{\eta_{1}\left(V_{1}(t)+V_{2}(t)\right)}{1+V_{1}(t)}-\eta_{2}\left(V_{3}(t)+V_{4}(t)\right) \\
& +\left(M_{0}+2+a\right)\left(\left|\varepsilon_{1}\right|+\left|\varepsilon_{2}\right|\right)\left(V_{1}(t)+V_{2}(t)+V_{3}(t)+V_{4}(t)\right)
\end{aligned}
$$

Observing that there exist positive constants $\mu_{1}, \mu_{2}, \mu_{3}$ and $\mu_{4}$ such that $\mu_{1}\left(V_{1}+V_{2}\right) \leq \Upsilon_{1} \leq \mu_{2}\left(V_{1}+V_{2}\right)$ and $\mu_{3}\left(V_{3}+\right.$ $\left.V_{4}\right) \leq \Upsilon_{2} \leq \mu_{4}\left(V_{3}+V_{4}\right)$ and that $\Upsilon_{i} \geq \Gamma_{i}(i=1,2)$, one obtains the existence of the functions $\varepsilon_{1}^{*}$ and $\varepsilon_{2}^{*}$ introduced in Theorem 2 such that, for $\left|\varepsilon_{1}\right|<\varepsilon_{1}^{*}\left(\Gamma_{1}(0), \Gamma_{2}(0)\right)$ and $\left|\varepsilon_{2}\right|<\varepsilon_{2}^{*}\left(\Gamma_{1}(0), \Gamma_{2}(0)\right)$, then

$$
\dot{V}(t) \leq-\frac{\eta_{1}\left(V_{1}(t)+V_{2}(t)\right)}{2\left(1+V_{1}(t)\right)}-\frac{\eta_{2}}{2} V_{3}(t)
$$

The rest of the proof of Theorem 1 remains unchanged.

\section{NUMERICAL SIMULATIONS}

To illustrate the merits of the proposed controller, we consider a numerical example inspired from the fluid flow system considered, e.g., in [4] [6]. It consists in an horizontal tube equipped with inlet and outlet fans and a mist injector. The mist injection is fixed and the control objective is to regulate the moisture at the output of the tube, that is, the ODE state $X$ is the moisture while the control $U$ is the fan power. A schematic view of the set-up is given in Fig. 1.

As studied in [4] [6], a stable first-order delay equation encapsulates the main features of the dynamics under consideration. Here, we consider that diffusion also occurs inside the tube. The moisture measured at the output thus satisfies (52) with $A=-1 / \tau_{0}$ and $B(\theta)=\theta / \tau_{0}$ in which the uncertain variable is the system gain (which slowly varies with thermodynamics conditions, and is then considered as uncertain). This system hence satisfies Assumptions 1-3 for any constant $K(\theta)<0$.

For simulation, we take ${ }^{1} a=1 / 6, \tau_{0}=9 \mathrm{~s}, \theta=0.08$ with

\footnotetext{
${ }^{1}$ Note that the convection speed is actually time-varying, but we neglect those variations here for the sake of simplicity. Also, diffusion is likely to be negligible compared to convection for this set-up in practice. Yet, for the sake of illustration, we consider the same scale for diffusion and convection coefficients in simulation.
} 


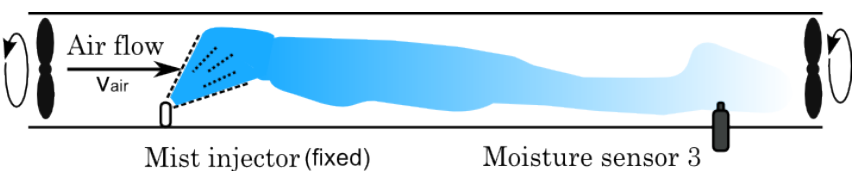

Fig. 1. Schematic view of the flowtube system under consideration as an illustrative example.

$\Pi=[0.05,0.11]$. We consider parameter PDE uncertainties as in (52) with $\varepsilon_{1}=0.1$ and $\varepsilon_{2}=-0.17$. We choose $K(\theta)=$ -40 . We compare closed-loop performances with a (similarly tuned) PI controller. Corresponding results are depicted in Fig. 2. One can observe that, when using the proposed adaptive controller, the obtained transient is much smoother. In particular, this controller enables to avoid oscillatory response which can be observed with a PI controller and are responsible for overshooting and actuator saturation. This is the main interest of the proposed controller.

\section{CONCLUSION}

In this paper, we proposed an adaptive controller enabling convection and diffusion compensation for a diffusive PDEODE cascade. Future works should focus on alleviating the assumption that the ODE state is measured, by studying a normal representation of the plant. A direct extension of the proposed work could be the design of a PDE-parameter adaptive controller. Also, counter-convection (that is, $a<0$ ) could be handled by a methodology similar to the proposed one by extending the design of [17]. However, as counterconvection has a potentially destabilizing effect, one cannot rely anymore on an open-loop observer for the PDE state, as done in this paper, and should assume that additional information is available for the PDE state. This is a direction of future investigation.

\section{REFERENCES}

[1] Z. Artstein. Linear systems with delayed controls: a reduction. IEEE Transactions on Automatic Control, 27(4):869-879, 1982.

[2] N. Bekiaris-Liberis and M. Krstic. Nonlinear Control Under Nonconstant Delays, volume 25. Society for Industrial and Applied Mathematics, 2013.

[3] D. Bresch-Pietri, J. Chauvin, and N. Petit. Adaptive control scheme for uncertain time-delay systems. Automatica, 48:1536-1552, 2012.

[4] D. Bresch-Pietri and K. Coulon. Prediction-based control of moisture in a convective flow. In Proc. of the European Control Conference, to appear, 2015.

[5] K. Gu and S. I. Niculescu. Survey on recent results in the stability and control of time-delay systems. Journal of Dynamic Systems, Measurement, and Control, 125:158, 2003.

[6] M. A. Hernandez Perez, E. Witrant, and O. Sename. A time-delay approach fro modeling and control of mist in a poiseuille flow. In Proc. of the European Control Conference, 2014.

[7] P. A. Ioannou and B. Fidan. Adaptive Control Tutorial. Society for Industrial Mathematics, 2006

[8] P. A. Ioannou and J. Sun. Robust Adaptive Control. Prentice Hall Englewood Cliffs, NJ, 1996.

[9] M. Jankovic. Recursive predictor design for linear systems with time delay. In American Control Conference, pages 4904-4909, 2008.

[10] M. Krstic. Boundary Control of PDEs: a Course on Backstepping Designs. Society for Industrial and Applied Mathematics Philadelphia, PA, USA, 2008.

[11] M. Krstic. Compensating actuator and sensor dynamics governed by diffusion PDEs. Systems and Control Letters, 58:372-377, 2009.

[12] M. Krstic, I. Kanellakopoulos, and P.V. Kokotovic. Nonlinear and Adaptive Control Design. John Wiley \& Sons New York, 1995.
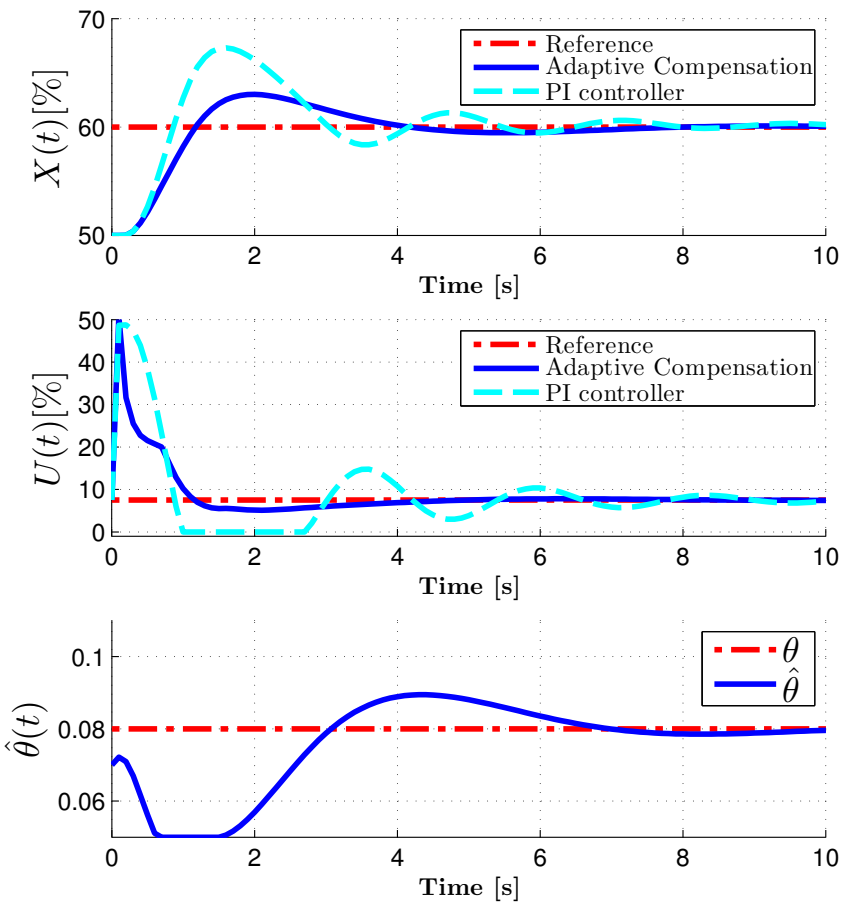

(a) Evolution of the moisture, the fan power and the parameter estimate.
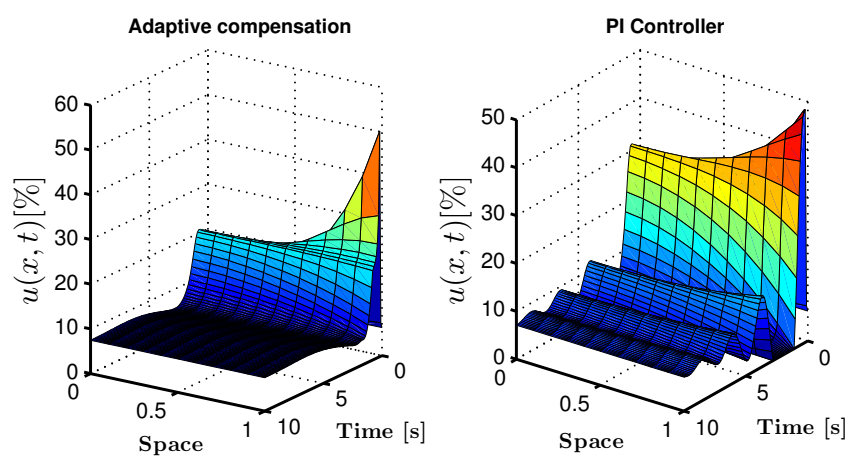

(b) Distributed input.

Fig. 2. Numerical results for the closed-loop dynamics of the moisture modeled by (52) with $A=-1 / \tau_{0}$ and $B(\theta)=\theta / \tau_{0}$, for two different controllers. The initial condition corresponds to a moisture (relative humidity) equilibrium of $50 \%$ that one wants to regulate to a new operating condition corresponding to $60 \%$ of moisture. The proposed adaptive controller has been implemented with a feedback gain $K(\hat{\theta})=-40$, an update gain $\gamma=60$ and a tuning parameter $b_{1}=10$. The PI controller has been implemented with a proportional gain $K_{P}=-40$ and an integral gain $K_{I}=-1$.

[13] A. Manitius and A. Olbrot. Finite spectrum assignment problem for systems with delays. IEEE Transactions on Automatic Control, 24(4):541-552, 1979

[14] W. Michiels and S. I. Niculescu. Stability and Stabilization of TimeDelay Systems. Society for Industrial and Applied Mathematics, 2007.

[15] J.-P. Richard. Time-delay systems: an overview of some recent advances and open problems. Automatica, 39(10):1667-1694, 2003.

[16] O. J. M. Smith. A controller to overcome dead time. ISA Journal, 6(2):28-33, 1959.

[17] G. A. Susto and M. Krstic. Control of PDE-ODE cascades with neumann interconnections. Journal of the Franklin Institute, 347(1):284314, 2010. 